\title{
Pre-treatment with mesenchymal stem cells reduces ventilator-induced lung injury
}

\author{
Laura Chimenti*,\#, Tomás Luque*, ${ }^{*}$, Maria R. Bonsignore\#, José Ramírez ${ }^{+, \S}$, \\ Daniel Navajas*, ${ }^{*, f}$ and Ramon Farrée, ${ }^{*, \S}$
}

ABSTRACT: Bone marrow-derived mesenchymal stem cells (MSCs) reduce acute lung injury in animals challenged by bleomycin or bacterial lipopolysaccaride. It is not known, however, whether MSCs protect from ventilator-induced lung injury (VILI).

This study investigated whether MSCs have a potential role in preventing or modulating VILI in healthy rats subjected to high-volume ventilation.

24 Sprague-Dawley rats (250-300 g) were subjected to high-volume mechanical ventilation $\left(25 \mathrm{~mL} \cdot \mathrm{kg}^{-1}\right)$. MSCs $\left(5 \times 10^{6}\right)$ were intravenously or intratracheally administered $(\mathrm{n}=\mathbf{8}$ each $) 30 \mathrm{~min}$ before starting over-ventilation and eight rats were MSC-untreated. Spontaneously breathing anesthetised rats $(n=8)$ served as controls. After $3 \mathrm{~h}$ of over-ventilation or control the animals were sacrificed and lung tissue and bronchoalveolar lavage fluid (BALF) were sampled for further analysis.

When compared with controls, MSC-untreated over-ventilated rats exhibited typical VILI features. Lung oedema, histological lung injury index, concentrations of total protein, interleukin$1 \beta$, macrophage inflammatory protein-2 and number of neutrophils in BALF and vascular cell adhesion protein-1 in lung tissue significantly increased in over-ventilated rats. All these indices of VILI moved significantly towards normalisation in the rats treated with MSCs, whether intravenously or intratracheally. Both local and systemic pre-treatment with MSCs reduced VILI in a rat model.

KEYWORDS: Acute lung injury, cell therapy, injurious ventilation, lung inflammation, lung oedema, mechanical ventilation

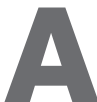
cute lung injury (ALI) and its most severe evolution, acute respiratory distress syndrome (ARDS), are devastating non-cardiac clinical diseases characterised by acute respiratory failure and bilateral pulmonary infiltrates consistent with oedema resulting from the disruption of the lung alveolar-capillary membrane barrier [1]. Despite extensive research into the pathogenesis and management of ALI and ARDS, mortality still remains high, at approximately $40 \%$ [2].

The status of ALI/ARDS patients can be aggravated by a significant side effect of mechanical ventilation, which is an essential vital support in these patients. Indeed, experimental and clinical evidence indicates that mechanical ventilation may exacerbate the patient's pre-existing condition or even damage healthy lungs, by inducing an inflammatory response that contributes to extrapulmonary organ dysfunction [3]. In fact, some features of ventilator-induced lung injury (VILI) resemble those of ALI/ARDS, and therefore act synergistically with them. VILI results from the action of mechanical forces on lung structures.
Over-stretching of alveolar walls causes endothelial and epithelial breaks and interstitial oedema. In particular, cyclic over-distension and the collapse/ re-opening of airway units with each breath are two key pathophysiological mechanisms leading to VILI [3]. To reduce the potential injurious effects of mechanical ventilation, protective ventilator strategies have been established and recommended in clinical guidelines [4].

Given the considerable health burden associated with ALI/ARDS, research on new therapeutic therapy approach recently proposed as a potential treatment for ALI/ARDS is based on the use of bone-marrow derived mesenchymal stem cells (MSCs) [5-7]. The rationale for applying adult stem cells of this type derives from their immunosuppressive and anti-inflammatory properties and their freedom from host rejection, allowing them to be used in allotransplantation $[8,9]$. Most of the experimental research carried out to date on the potential therapeutic function of MSCs in ALI/ ARDS has been focused on the primary causes of lung injury. Specifically, data obtained in different strategies for this syndrome is very active. A cell

\section{AFFILIATIONS}

*Unitat de Biofísica i Bioenginyeria, Facultat de Medicina, Universitat de Barcelona,

+Departament d'Anatomia Patologica, Hospital Clinic, Facultat de Medicina Universitat de Barcelona

§Institut d'Investigacions Biomèdiques August Pi i Sunyer, and ${ }^{f}$ Institut de Bioingenyeria de Catalunya, Barcelona, and "CIBER de Enfermedades Respiratorias, Bunyola, Spain. \#Biomedical Dept of Internal and Specialist Medicine (DIBIMIS), Section of Pneumology, University of Palermo, Palermo, Italy.

CORRESPONDENCE

R. Farré

Unitat de Biofísica i Bioenginyeria

Facultat de Medicina

Casanova 143

08036 Barcelona

Spain

E-mail: rfarre@ub.edu

Received:

Sept 062011

Accepted after revision:

Feb 142012

First published online:

March 222012 
studies carried out on cell cultures, rodent models and ex vivo human lungs have shown that MSCs alleviate the severity of acute lung injury caused by bleomycin [10] or bacterial lipolysaccharide (LPS) [11, 12] and act as potent antimicrobial treatment [13]. Whereas some authors injected the MSCs intravenously, others instilled the cells directly into the trachea $[10,11,14]$, and apparently positive effects were achieved by both means.

However, whether MSCs could be effective for reducing or modulating VILI remains to be elucidated. Since VILI is characterised by lung inflammation and MSCs exhibit antiinflammatory properties, the hypothesis of this work was that pre-treatment with these adult stem cells could attenuate VILI. Accordingly, the aim of this experimental research was to study the potential therapeutic effect of MSCs in an acute animal model of VILI induced by high tidal volume ventilation. Given that the best route for MSCs application for treating lung injury is unknown, we assessed whether the intravenous or intratracheal administration of these stem cells would result in the same potential reduction of VILI.

\section{METHODS \\ Animals}

The study was carried out on 38 pathogen-free male SpragueDawley rats (250-300 g; Criffa, Lyon, France) that were housed in light-dark cycle-regulated, air-conditioned $\left(23^{\circ} \mathrm{C}\right)$ and airhumid $(60 \%)$ animal quarters and had free access to standard food pellets (A04; Panlab, Barcelona, Spain) and tap water. This experimental work was approved by the Ethical Committee for Animal Research of the University of Barcelona.

\section{MSCs}

Lewis rat marrow stromal cells kindly provided by the Tulane Center for Gene Therapy (Tulane University, LA, USA) were used in this study. These cells are well characterised [15] and used in several published reports, being positive/negative for relevant MSC surface markers and able to develop osteogenic, chondrogenic and adipogenic differentiation. Cells (passage 611) were cultured in MEM- $\alpha$ (Gibco; Gaithersburg, MD, USA) supplemented with $20 \%$ of fetal bovine serum (FBS; Hyclone Cell Culture, Logan, UT, USA), $1 \%$ of antibiotic-antimycotic $\left(10,000 \mathrm{U} \cdot \mathrm{mL}^{-1}\right.$ penicillin $\mathrm{G}$ sodium, $10,000 \mu \mathrm{g} \cdot \mathrm{mL}^{-1}$ streptomycin sulphate, $25 \mu \mathrm{g} \cdot \mathrm{mL}^{-1}$ amphotericin B; Gibco) and $2 \%$ of L-glutamine (200 $\mathrm{mM}$ in $0.85 \% \mathrm{NaCl}$; Gibco). Cells were grown in an incubator $\left(37^{\circ} \mathrm{C}, 5 \% \mathrm{CO}_{2}, 100 \%\right.$ humidity). Subconfluent cells were dissociated with $0.25 \%$ trypsin and $1 \mathrm{mM}$ EDTA in Hanks' balanced salt solution (Gibco) and subcultured at low density in new culture flasks.

\section{Administration of MSCs and induction of VILI}

Animals were hydrated with subcutaneous injection of physiological saline solution and intraperitoneally anesthetised with $10 \%$ urethane $(5 \mathrm{~mL}$ per $300 \mathrm{~g}$ body weight; Sigma, St Louis, $\mathrm{MO}, \mathrm{USA})$. The rectal temperature of the rats was maintained within the range of $36.5-37.5^{\circ} \mathrm{C}$ throughout the procedure. The animals were randomly assigned to four groups ( $n=8$ each). One control group was kept under spontaneous breathing for the entire duration of the experiment, while the other three groups of rats were tracheostomised and intubated (cannula 16GA BD Adsyte Pro, Becton Dickinson; Madrid, Spain). Subsequently, one group was kept under spontaneous breathing and the animals from another two groups were subjected to the following interventions: 1) injection of $5 \times 10^{6}$ MSCs diluted in $0.3 \mathrm{~mL}$ of sterile Dulbecco's phosphate-buffered saline (DPBS) through the penile vein; 2) intratracheal instillation of $0.3 \mathrm{~mL}$ of DPBS containing $5 \times 10^{6}$ MSCs through the intubation cannula. During the intratracheal instillation experiments, the rats were maintained in a head-up tilted position to prevent MSC spillage. After $30 \mathrm{~min}$ of spontaneous breathing, the three groups of intubated rats were subjected to high-volume ventilation to induce VILI. To this end, the rats were paralysed by infusion of pancuronium bromide $\left(0.4 \mathrm{mg} \cdot \mathrm{kg}^{-1}\right.$ body weight; P1918, Sigma) into the penile vein and rapidly connected to a rodent ventilator (Harvard Apparatus, Model 683; South Natick, MA, USA). The animals were ventilated with room air at a rate of 80 breath$\mathrm{s} \cdot \mathrm{min}^{-1}$ using a high tidal volume of $25 \mathrm{~mL} \cdot \mathrm{kg}^{-1}$. After $3 \mathrm{~h}$ of high-volume ventilation (or spontaneous breathing in the control group) the rats were sacrificed by exsanguinations.

\section{Obtaining and processing bronchoalveolar lavage fluid}

After exsanguination, the left main bronchus was tied with a string at the left hilum. Bronchoalveolar lavage fluid (BALF) was obtained from the right lung by connecting a syringe to the cannula placed into the trachea and then flushing through it $3 \mathrm{~mL}$ of sterile phosphate-buffered saline four times. BALF recovery was always greater than $85 \%$. Cells in the BALF were counted on a haemocytometer (Neubauer, Marienfeld, LaudaKönigshofen, Germany) from slides prepared by cytocentrifugation (Shandon Cytospin 4, Thermo Electron Corporation, Marietta, OH, USA) and Diff-Quick staining (Pancreac Quimica SAU; Castellar del Vallès, Spain). For each rat, approximately 500 cells were counted. Moreover, the BALF was centrifuged at $1,300 \times g$ for $10 \mathrm{~min}$ and the supernatant stored at $-80^{\circ} \mathrm{C}$ for subsequent analysis.

\section{Lung wet/dry weight ratio}

The left lungs were dissected, immediately after exsanguination, for oedema assessment and the wet weight recorded. The lungs were then placed in an incubator at $55^{\circ} \mathrm{C}$ for $24 \mathrm{~h}$, and the dry weight was determined.

\section{Histological examination}

The right lungs were removed and one lobe was fixed in $10 \%$ paraformaldehyde. The histological analysis of excised lung tissue was carried out by two independent experts, blinded to the treatment, using $4-\mu \mathrm{m}$ sections stained with haematoxylin and eosin (H\&E). A computerised analysis was carried out by digitally capturing the image of each whole H\&E-stained section with a Leica DMD108 Digital Microimaging Device (Leitz Microsystems; Wetzlar, Germany). A threshold analysis of whole lung sections, manually excluding non-parenchymal structures (e.g. large blood vessels, airway structural elements and connective structures), was performed by using the Metamorph image analysis software (Molecular Devices; Sunnyvale, CA, USA) by a blinded investigator. To this end, red-green-blue colour images were split into individual colour channels and analyses were performed using the green channel. The threshold was then adjusted to the highest point that would highlight all areas of the parenchyma while excluding background. The threshold area of total parenchyma was measured and recorded. Then the threshold was reset to highlight only areas of high pixel density correlating with lung damage (damage threshold), as 

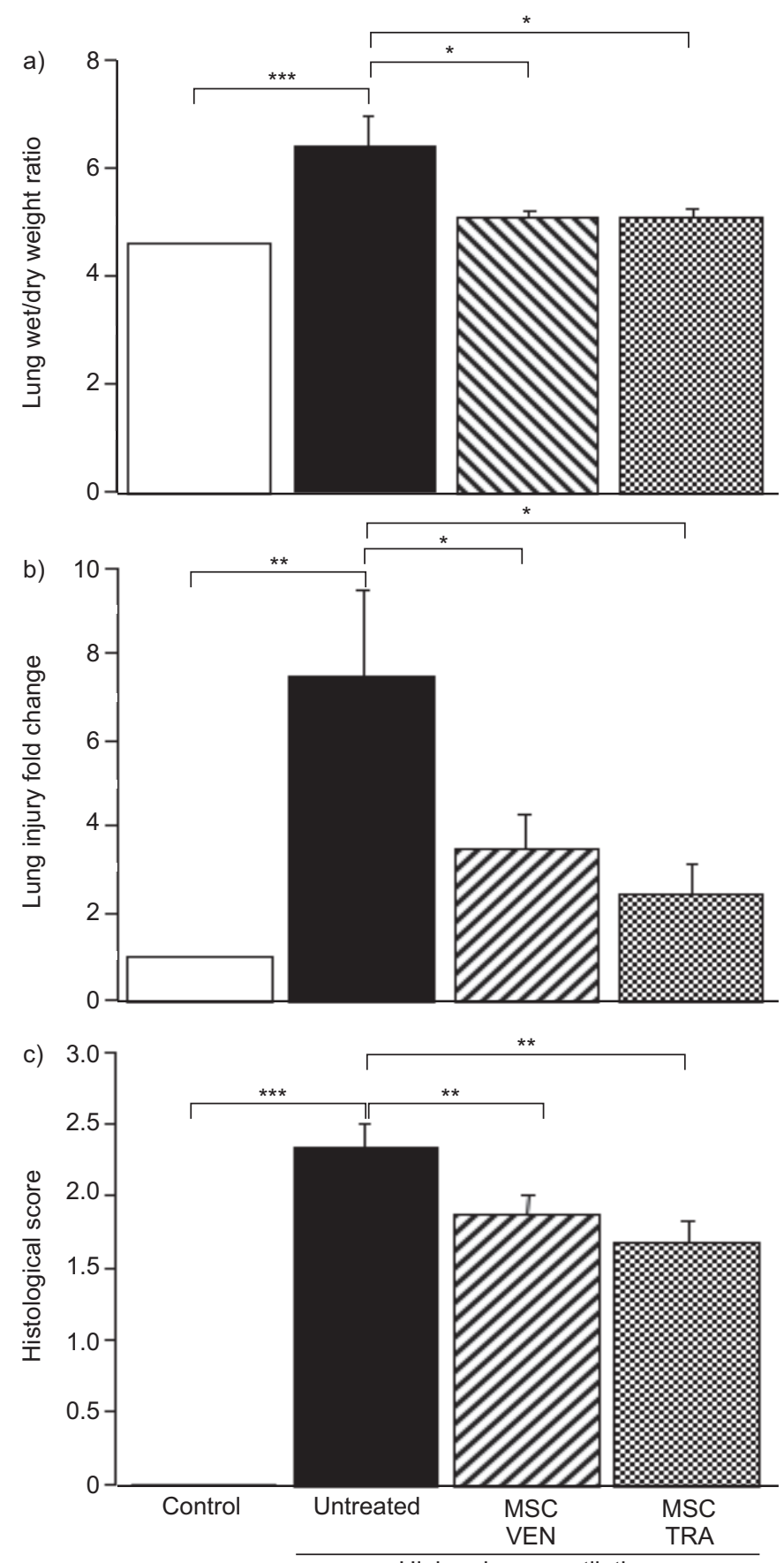

High-volume ventilation

FIGURE 1. Lung oedema, quantified by the a) wet/dry weight ratio, b) histological index of lung injury and c) histological score in control rats and in rats subjected to high-volume ventilation with or without instillation of mesenchymal stem cells (MSC) intravenously (VEN) or intratracheally (TRA). Data are presented as mean \pm SEM. *: $p<0.05 ; * *: p<0.001 ; * * *: p<0.0001$

determined by microscopic regional image analysis. Equal threshold values were applied to all the images analysed in all the groups. A lung injury index was calculated as the ratio of damaged area relative to total parenchyma area, and this was reported as fold increase over control lungs (non-ventilated rats) [16]. In addition, a pathologist blinded to the rat's experimental conditions scored the right lung tissue preparations according to the following scale: 0: normal lung; 1: septal congestion; 2: septal inflammatory infiltrates; 3 : alveolar haemorrhage and/or hyaline membranes.

\section{MSC staining}

MSCs $\left(5 \times 10^{6}\right)$ were fluorescent-labelled with Vybrant -DiI $\left(1,1^{\prime}-\right.$ Dioctadecyl-3,3,3', 3'-tetramethylindocarbocyanine perchlorate) cell labelling solution (Molecular Probes, Carlsbad, CA, USA) for $20 \mathrm{~min}$ at $37^{\circ} \mathrm{C}$ and then washed and resuspended with DPBS. After labelling, MSCs were intratracheally or intravenously applied in six additional rats as previously described. Right lungs from MSC pre-treated rats subjected to the high-volume ventilation were frozen in Tissue-Tek (Sakura Finetechnical, Tokyo, Japan) optical cutting temperature (OCT) compound by immersion in liquid nitrogen. Cryostat sections (10 $\mu \mathrm{m}$ thick) were adhered to glass slides previously coated with poly-L-lysine, fixed in formalin for $10 \mathrm{~min}$ and analysed by a fluorescence microscope (Eclipse Ti; Nikon Instruments Inc, NY, USA)

\section{Immunohistochemistry}

Vascular cell adhesion protein (VCAM)-1 expression in lung tissues was assessed by using a monoclonal antibody (H-276: sc8304; Santa Cruz Biotechnology Inc., CA, USA) following the manufacturer's protocol for paraffin-embedded tissues. Immunoreactivity was revealed by the labelled streptavidin biotin alkaline-phosphatase technique (Dako Cytomation, Glostrup, Denmark). Lung sections were counterstained with haematoxylin and read by an investigator blind to the group of the sample. VCAM-1 quantification was carried out by using a computerised analysis, as previously reported, and calculated as the ratio of VCAM-1 expression area relative to total parenchyma area.

\section{RNA extraction and real-time PCR}

Total RNA from lung tissue was isolated with the Prime script RT reagent kit (Takara Bio Inc, Otsu, Japan). Total RNA was then quantified and quality evaluated by using a spectrophotometer (Biophotometer; Eppendorff, Hamburg, Germany). A total of $100 \mathrm{ng} \cdot \mu \mathrm{L}^{-1}$ RNA was used for reverse transcriptase PCR using the Taqman Master Mix (Applied Biosystems, Foster City, CA, USA) according to the manufacturer's instructions. Quantitative real time-PCR (Applied Biosystems) was employed to analyse the tissue distributions using the gene-specific primers (IDT; Integrated DNA Technologies, Germany), interleukin (IL)-1 receptor antagonist (IL-1RA) primer 1: 5'-AGCGGATGAAGGTAAAGCG-3', primer 2: 5'-CTGTGCCTGTCTTGTGTCA-3'; keratinocyte growth factor (KGF) primer 1: 5'-CACAATTCCAACTGCCACAG-3' primer 2: 5'GGATTGACAAACGAGGCAAAG-3'; hepatocyte growth factor (HGF) primer 1: 5'-CAAACTAACCATCCACCCTACT-3', primer 2: 5'-ATTGCCCTATTTCCCGTTGT-3'. The expression level of glyceraldehydes-3-phosphate dehydrogenase was used as the internal control; primer 1: 5'-GTAACCAGGCGTCCGATAC-3', primer 2: 5'-GTTCTAGAGACAGCCGCATC-3'.

\section{Cytokine and protein measurements}

Total protein concentration in BALF was quantified using the Micro BCA protein assay kit (Pierce, Rockford, IL, USA). The concentration of IL-1 $\beta$ and macrophage inflammatory protein-2 (MIP-2) in BALF was determined by ELISA (Quantikine ELISA Kit; R\&D Systems, Minneapolis, USA). 

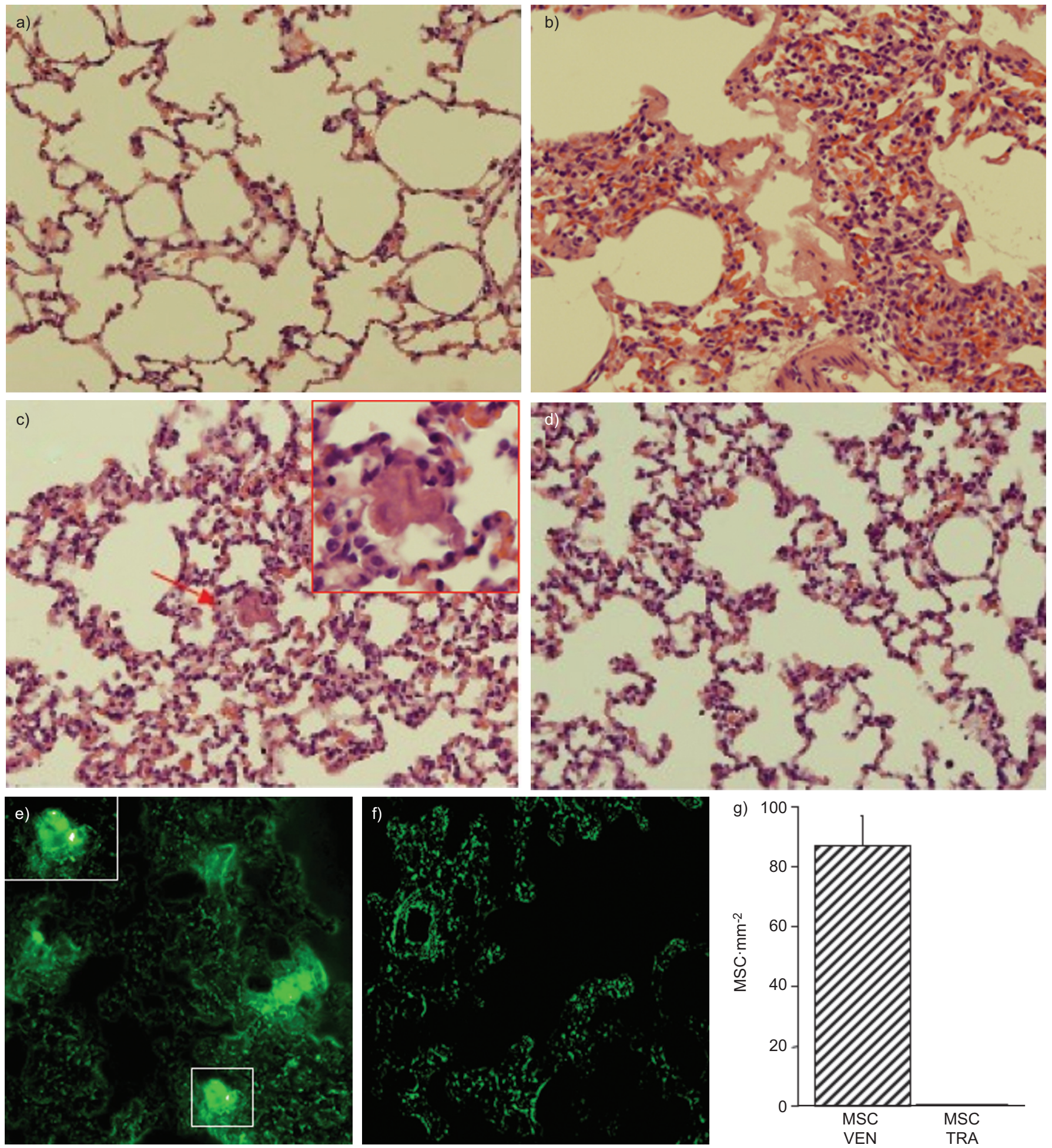

FIGURE 2. Representative images of a-d) haematoxylin and eosin-stained and e, f) Vybrant Dil-stained lung tissue sections. In rats subjected to high-volume ventilation pretreated with mesenchymal stem cells (MSC) either intravenously (VEN; c) or intratracheally (TRA; d), lung injury was notably reduced compared with over-ventilated rats (b). The arrow shows MSCs localised in the tissue sections. Original magnification $\times 200$. g) Quantification of the MSCs localised in lung tissue. Data are presented as mean \pm SEM.

\section{Statistical analysis}

Results are reported as mean \pm SEM. ANOVA with Fisher's protected least significant difference as a post hoc analysis was used for multigroup comparisons (StatView 5.0.1; Abacus Concept, Berkeley, CA, USA). Statistical significance was established as $\mathrm{p}<0.05$. 

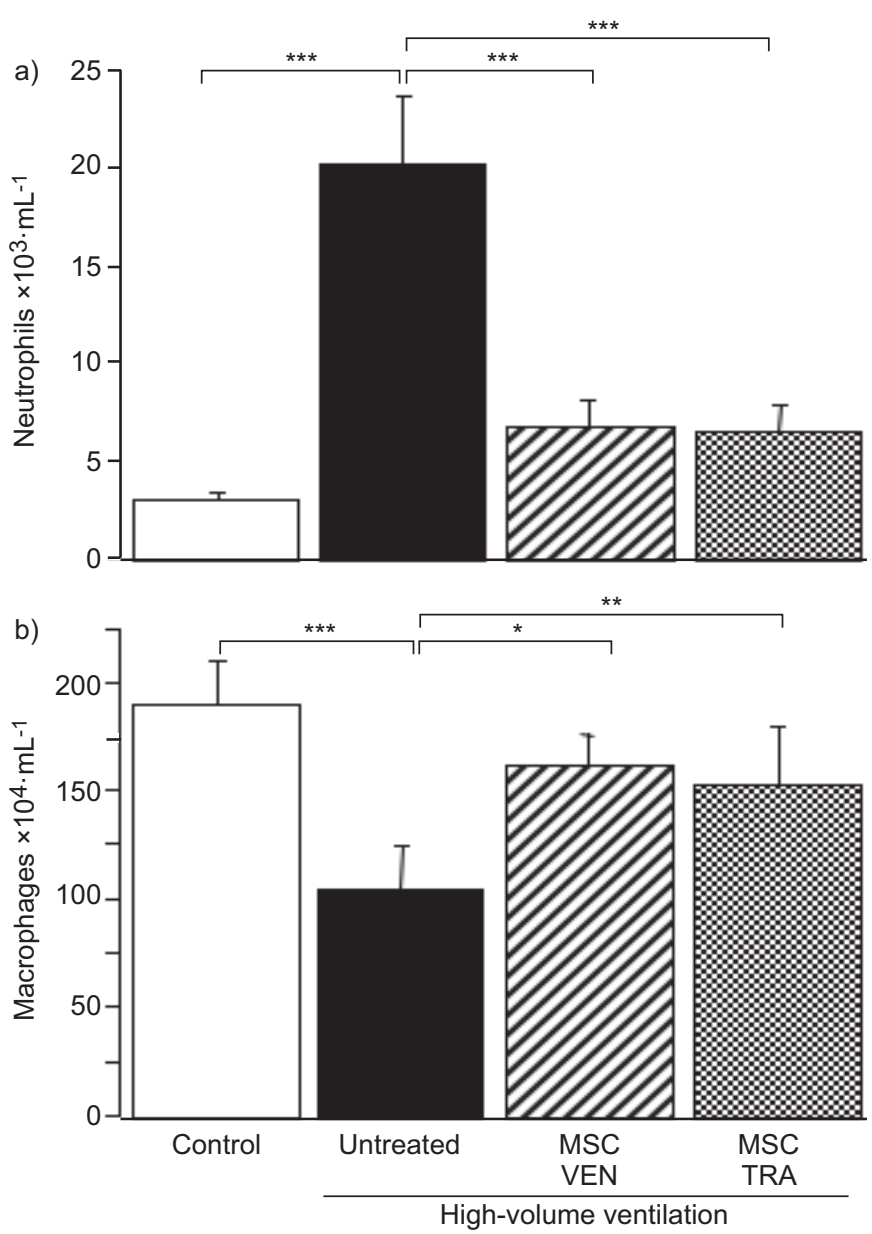

FIGURE 3. Absolute a) neutrophil and b) macrophage counts in the bronchoalveolar lavage fluid in control rats and in rats subjected to high-volume ventilation, with or without instillation of mesenchymal stem cells (MSC) intravenously (VEN) or intratracheally (TRA). Data are presented as mean \pm SEM. $*: p<0.05 ; * *: p<0.01 ; * * *: p<0.001$.

\section{RESULTS}

Lung oedema assessed by the wet/dry weight ratio method was significantly greater in animals subjected to over-ventilation than in controls. In over-ventilated animals, pre-treatment with MSCs reduced oedema to values very close to those of controls. A similar improvement was found for intratracheal and intravenous administration route of MSCs (fig. 1a). The results obtained from the two histological analyses of lung tissues, the computerised lung injury index and the visual score provided consistent results (fig $1 \mathrm{~b}$ and c). Whereas lung injury was significantly detected in the over-ventilated MSCuntreated animals (figs 1 b, c and 2b), in the rats pre-treated with MSCs, regardless of the administration route, lung injury was considerably reduced (figs $1 b, c, 2 c$ and $d$ ). Figure $2 c-g$ indicates that MSCs were localised in the tissue sections only in case that these cells were applied intravenously.

Figure 3 shows the results of cell counting in BALF: neutrophils significantly increased and macrophages significantly decreased in the over-ventilated animals, while pre-treatment with MSCs, either intravenously or intratracheally, reduced neutrophils and increased macrophages (fig. $3 a$ and b, respectively). Data in figure 4 indicate that MSCs were localised in the BALF only in the cases that these cells were applied intratracheally. Total cell counts in BALF were almost normalised in the over-ventilated animals that were subjected to either intravenous or intratracheal pre-treatment with MSCs $(1.70 \pm$ $0.14 \times 10^{4}$ cells $\cdot \mathrm{mL}^{-1}$ and $1.72 \pm 0.27 \times 10^{4}$ cells $\cdot \mathrm{mL}^{-1}$, respectively) whilst they decreased in the BALF of over-ventilated rats compared with controls $\left(1.28 \pm 0.22 \times 10^{4}\right.$ cells $\cdot \mathrm{mL}^{-1}$ and $1.94 \pm 0.19 \times 10^{4}$ cells $\cdot \mathrm{mL}^{-1}$, respectively; $\left.\mathrm{p}<0.05\right)$.

Figure $5 \mathrm{a}-\mathrm{c}$ shows that the concentrations of total proteins, pro-inflammatory cytokine IL-1 $\beta$ and MIP-2 in BALF were significantly higher in MSC-untreated animals subjected to high-volume ventilation than in controls. Application of MSCs, either intravenously or intratracheally, in over-ventilated rats markedly reduced the amount of total proteins, IL-1 $\beta$ and MIP-2 in BALF (fig. 5a--c). Figure $5 \mathrm{~d}$ also shows that real-time PCR showed a significant increase in mRNA levels of IL-1RA in lungs of MSC pre-treated groups compared to controls and to animals subjected to high-volume ventilation. mRNA analysis of HGF and KGF in the lung tissues did not reveal any significant difference among the groups (data not shown).

Expression of VCAM-1, evaluated by immunohistochemical examination of lung tissue, was found to be greater in the overventilated group (fig. 6b), whilst pre-treatment with MSCs, either intravenously or intratracheally, decreased VCAM-1 to levels close to control values (fig. 6a, c, d and e).

\section{DISCUSSION}

The results obtained in this experimental high-volume ventilation model confirm the hypothesis that MSCs prevent lung injury induced by high-amplitude mechanical ventilation. It was also found that the early potential therapeutic effect of MSCs was similar for both local (intratracheal) and systemic (intravenous) infusion. MSCs were localised in lung tissue only in case that these cells were injected intravenously, whilst MSCs applied intratracheally were only found in BALF.

This study was carried out with a common rat model of VILI, with typical magnitude of overstretch $\left(25 \mathrm{~mL} \cdot \mathrm{kg}^{-1}\right)$ and duration of the injurious mechanical stimulus ( $3 \mathrm{~h}$ ) [17]. The animals subjected to lung over-distension exhibited the expected pattern of VILI; namely loss of lung barrier integrity and lung inflammation. Lung oedema, resulting from increased permeability of the alveolocapillary barrier, augmented from a normal value of lung wet/dry weight ratio to a figure typically found in animals subjected to over-ventilation [17]. Accordingly, the histopathological tissue analysis showed considerable lung injury in the animals receiving high-volume ventilation. Moreover, cell and protein analysis of BALF showed a clear pattern of VILI in the animals subjected to overstretch: 1) increased number of neutrophils, caused by augmented endothelial and epithelial permeability [1, 18]; 2) reduced counts of alveolar macrophages, consistent with the rapid decline in BALF macrophages found in other works in response to high-volume ventilation [19] and attributed to their fast inflammatory activation [20]; 3) increased total protein concentration, a hallmark of leakage in the lung barrier [1, 17]; 4) augmented concentration of cytokines MIP-2 and IL-1 $\beta$, among the most biologically active cytokines in the lungs of patients with ALI [21, 22]; 5) in lung tissue, increased VCAM-1 expression, 


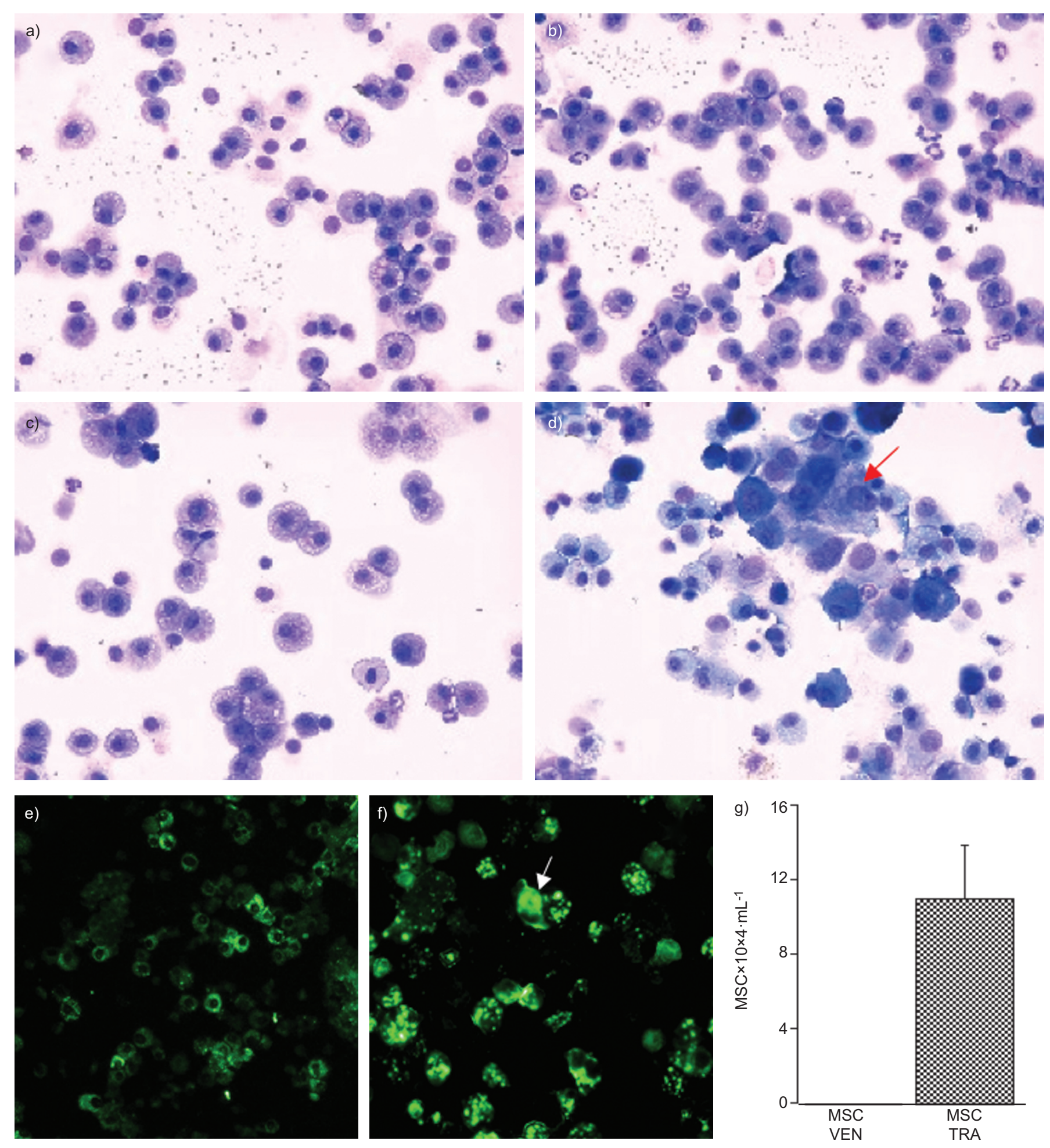

FIGURE 4. Images of a-d) citospin preparations and e, f) Dil staining of bronchoalveolar lavage fluid (BALF) from a) control rats and rats subjected to high-volume ventilation b) without or c, e) with administration of mesenchymal stem cells (MSC) intravenously (VEN) or $\mathrm{d}$, f) intratracheally (TRA). The arrows show the presence of MSCs in BALF of rats intratracheally instilled with MSCs. Original magnification $\times 400$. g) Number of MSCs recovered in BALF. Data are presented as mean \pm SEM.

having an important role in neutrophil and lymphocyte sequestration in VILI [23]. Taken together, these common biomarkers of lung injury indicate that the animals subjected to high-volume ventilation and not treated with MSCs developed VILI.
Whereas some studies on the protective effects of adult stem cells in lung injury have been carried out using bone marrowderived mononuclear cells, including both mesenchymal and hematopoietic stem cells [4-6], in this work we focused on MSCs 

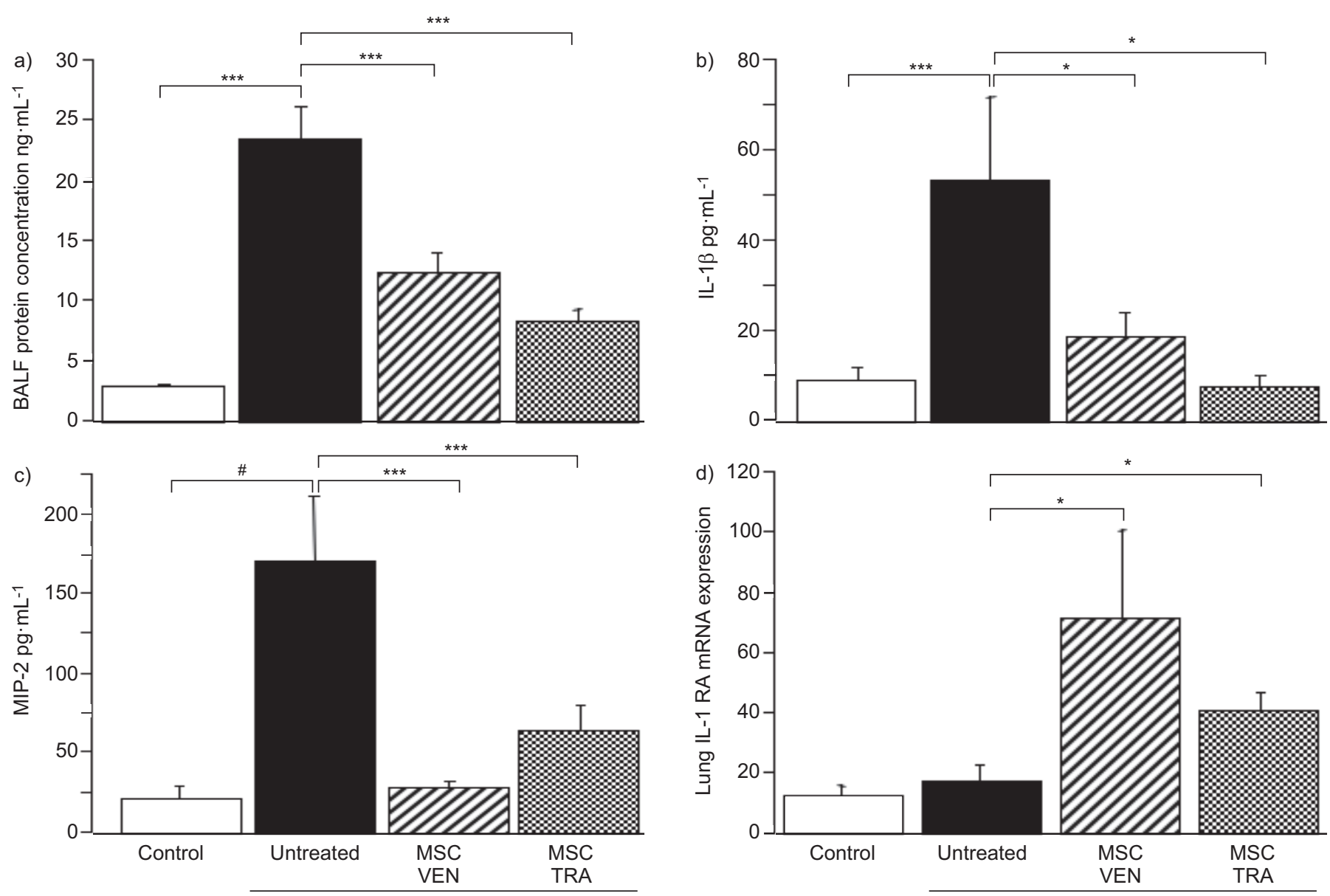

High-volume ventilation

High-volume ventilation

FIGURE 5. a) Total proteins, b) cytokine interleukin (IL)- $\beta 1$ and c) macrophage inflammatory protein-2 (MIP-2) concentrations in bronchoalveolar lavage fluid (BALF) in control rats and in rats subjected to high-volume ventilation with or without instillation of mesenchymal stem cells (MSC) intravenously (VEN) or intratracheally (TRA). d) IL-1 receptor antagonist (IL-1RA) mRNA expression in lung tissue. Data are presented as mean \pm SEM. ${ }^{*}: p<0.05 ;{ }^{* *} p<0.001 ;{ }^{*}: p<0.0001$.

exclusively. We used well-characterised MSCs [15] and applied a number of cells in keeping with the different doses reported in the literature when these stem cells were applied, either intravenously or intratracheally, in rats and mice [10, 24].

Pre-treatment with MSCs was effective in significantly reducing the development of VILI in the rats subjected to over-ventilation. Our results clearly indicate that all the variables assessed in the animals pre-treated with MSCs exhibited a tendency to recover the control values, i.e. oedema and histological index of injury in lung; neutrophils and macrophages cell counts and biomolecules concentration in BALF and in lung tissue increased mRNA IL-1RA expression. Taken together, these data provide solid evidence that the local inflammatory process and the associated loss of alveolocapillary membrane integrity induced by highvolume ventilation were considerably reduced. The effectiveness of MSCs in minimising the manifestation of VILI was in agreement with findings observed in bleomycin or LPS-induced lung injury $[10,11]$.

Data from bleomicyn or LPS in ALI models also suggest that the protective effect of MSCs may be similar, regardless of the route of injection [10,11, 14]. Our group is the first to show evidence, in a single study, that either intravenous or intratracheal administration of MSCs may result in the same potential benefits in the reduction of VILI. We observed that MSCs injected via the intra-alveolar route were recovered in the BALF, highlighting that this injection method would maximise cell delivery in the lung and directly reach the injury site. When MSCs were administered via the systemic route, however, they became localised in the alveolar region, confirming the finding that MSCs arrive first at the pulmonary microcirculation. Their retention in the lung may be facilitated by their large size with respect to the capillary diameter, and by the expression of adhesion molecules, such as VCAM- 1 and P-selectin [25]. However, the fact that systemic application of MSCs can be as effective as local application in protecting against lung injury might have some practical clinical relevance as intravenous administration is much simpler than intratracheal administration in patients, even those who are intubated and mechanically ventilated.

Since several studies have shown that pulmonary engraftment of MSCs in the lungs does not occur very easily, the ability of MSCs to reduce lung injury in different ALI models is currently attributed to a paracrine effect [26, 27]. Irrespective of the route of delivery, MSCs may reduce lung injury and promote repair by interacting with injured resident alveolar 

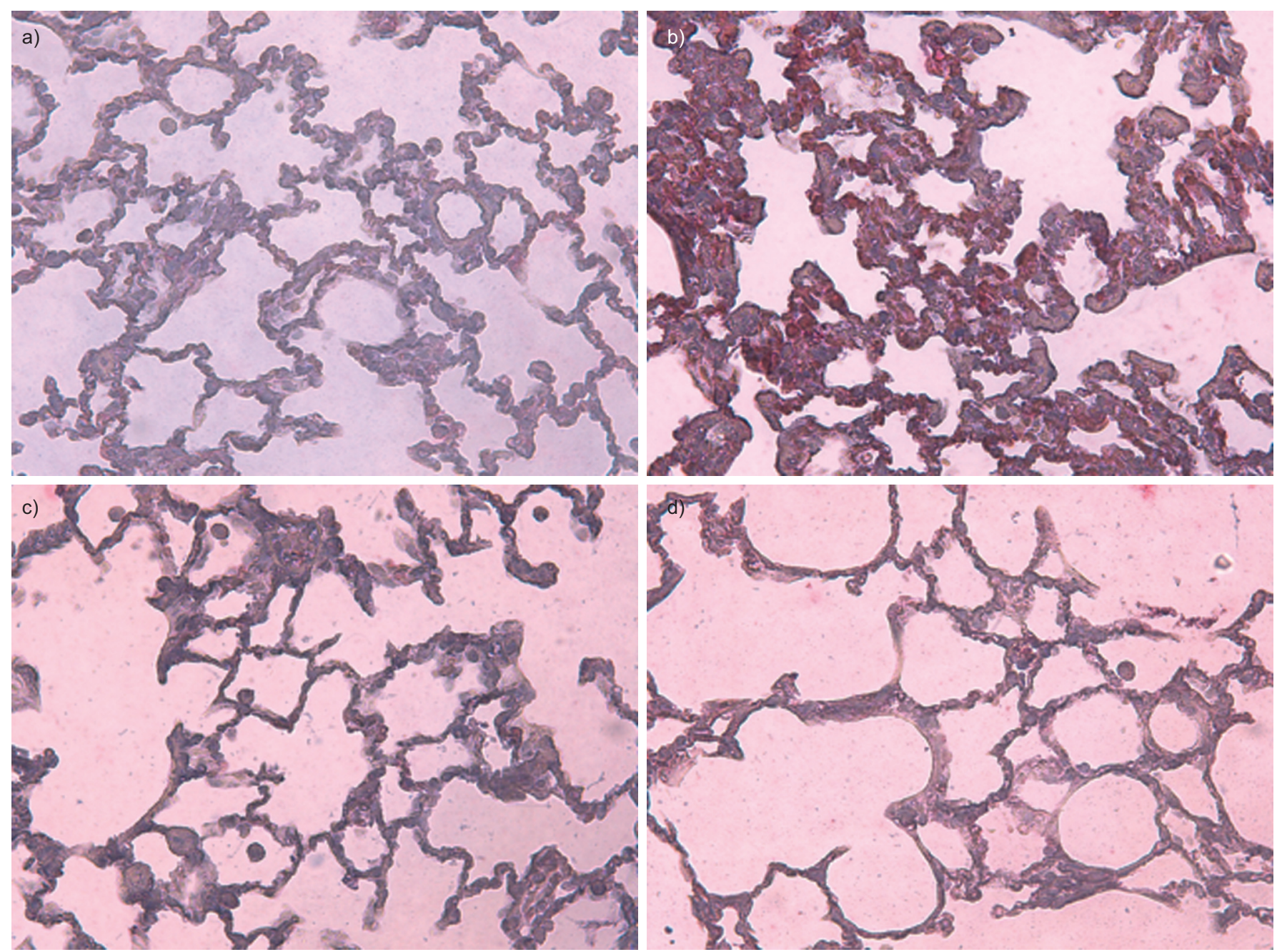

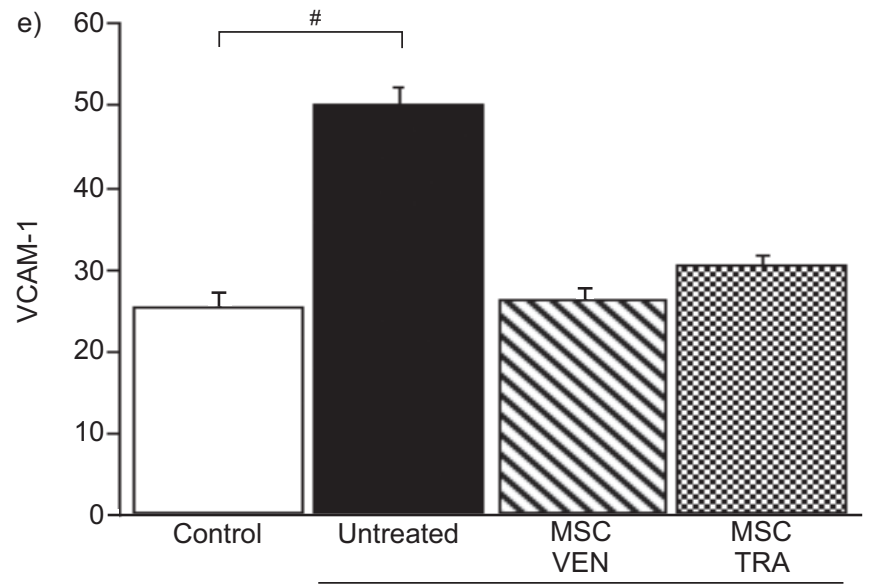

High-volume ventilation epithelial or lung endothelial cells, or by modulating the responses of neutrophils, monocytes, activated macrophages, lymphocytes with the release of several secreted factors such as angiopoietin (Ang)-1, prostaglandin $\mathrm{E}_{2}\left(\mathrm{PGE}_{2}\right)$, IL-1RA, transforming growth factor- $\beta$ (TGF- $\beta$ ). Furthermore, MSCs may secrete growth factors, such as vascular endothelial growth factor (VEGF), KGF and HGF, which have cytoprotective
FIGURE 6. a-d) Representative images of vascular cell adhesion protein (VCAM)-1 immunohistochemical staining. In rats subjected to high-volume ventilation pre-treated with mesenchymal stem cells (MSC) either intravenously (VEN; c) or intratracheally (TRA; d), VCAM-1 expression greatly decreased compared with over-ventilated rats (b). e) Data are presented as mean \pm SEM. \#: $p<0.0001$

and reparative properties $[5,26,27]$. In our acute study, we observed increased expression of IL-1RA mRNA in lung tissue when over-ventilated animals were pre-treated with MSCs, irrespective of the administration route, while no major changes in the mRNA expression of KGF or HGF were detected among all the groups after $3 \mathrm{~h}$ of injurious ventilation. In an LPSinduced ALI model, a significant decrease in pro-inflammatory 
cytokines (tumour necrosis factor (TNF)- $\alpha$, MIP-2) and an increase in anti-inflammatory cytokines, including IL-10 and IL-13, were observed when MSCs were given via the intratracheal route [11]. Similar results were achieved when the culture medium of MSCs was administered for treatment of LPS-induced ALI in an ex vivo perfused human lung [14]. Further evidence that paracrine factors released by MSCs or the conditioning medium play a beneficial role in the reversal of ALI comes from studies on perinatal hyperoxic models of lung injury [28].

Our study was conceived to test the hypothesis that MSCs may reduce VILI. Although the experimental design was close to that of conventional studies using rodent VILI models and MSCs infusion, the present work shows some limitations because not all the possible ranges of variables were investigated. To induce VILI we did not investigate different ventilator settings (amplitude of tidal volume, effect of positive end-expiratory pressure (PEEP) or pressure- rather than volume-controlled ventilation). A previous study, however, showed that these variables can modulate the magnitude of VILI but not its mechanisms and consequences (lung membrane leakage and inflammation) [29]. A further limitation, in common with most research studies on treatment with MSCs is about the timing of MSCs instillation with respect to the start of the injurious stimulus, in our study, mechanical ventilation. Whether the protective effects of MSCs could depend on these parameters remains to be elucidated. The effects could be different, in fact, if MSCs were applied as a preventive treatment and a different concentration with respect those ones we used, or once lung injury has been well established. Finally, we focused on the early effects of VILI $(3 \mathrm{~h})$. Extending the duration of the experiments would allow assessing the study in more details, specifically the expression and role played by different biomarkers in the mid- and long-term. Accordingly, the potential therapeutic effect of MSC in chronic models of VILI requires further investigation. In particular, future experiments assessing the effect of MSCs on already well established VILI are of major interest to better approach the clinical problem. Regardless of its limitations, this is the first study, to our knowledge, that provides proof for the concept that both local and systemic pre-treatment with MSCs could play a role in protecting the lungs from the injuries caused by mechanical ventilation of high amplitude.

MSCs are emerging as a potential therapeutic tool in patients. In fact, a considerable number of clinical trials using this type of cells have recently been completed, or are ongoing, for various diseases, including acute myocardial infarction, ischaemic heart failure, renal failure, multiple sclerosis, aseptic necrosis of the hip, inflammatory bowel disease, osteogenesis imperfecta and also respiratory diseases such as idiopathic pulmonary fibrosis and chronic obstructive pulmonary disease [5]. To date, most of the research in MSCs has been carried out with cells obtained from the bone marrow. It is expected, however, that MSCs obtained from more accessible niches, for instance umbilical cord blood and, particularly, adipose tissue, could facilitate both the heterologous and autologous infusion of MSCs. Although MSCs from different niches do show some differences, their main biological features are similar. In fact, some studies have shown that MSCs derived from adipose tissue and from bone marrow perform similarly, for instance for ischemic stroke therapy [30]. Accordingly, both types of adult stem cells are currently used in clinical trials [31]. It is therefore likely that future research could prove that MSCs obtained from sites other than bone marrow could also be helpful in alleviating VILI and, more generally, ALI/ARDS.

\section{SUPPORT STATEMENT}

This research was supported in part by the Ministerio de Ciencia e Innovación (SAF2008-02991 and PI081908). L. Chimenti is the recipient of a European Respiratory Society and Marie Curie Joint Research Fellowship (MC 1636-2010). The research leading to these results has received funding from the European Respiratory Society and the European Community's Seventh Framework Programme FP7/20072013 - Marie Curie Actions under grant agreement RESPIRE, PCOFUND-GA-2008-229571.

\section{STATEMENT OF INTEREST}

None declared.

\section{ACKNOWLEDGEMENTS}

The authors wish to thank I. Almendros, R. Nieto and M.A. Rodríguez (Unit of Biophysics and Bioengineering, Faculty of Medicine, University of Barcelona, Barcelona, Spain) for their valuable technical help. The mesenchymal stem cells employed in this work were kindly provided by the Tulane Center for Gene Therapy, New Orleans, LA, USA.

\section{REFERENCES}

1 Ware LB, Matthay MA. The acute respiratory distress syndrome. N Engl J Med 2000; 342: 1334-1349.

2 Matthay MA, Zimmerman GA. Acute lung injury and the acute respiratory distress syndrome: four decades of inquiry into pathogenesis and rational management. Am J Respir Cell Mol Biol 2005; 33: 319-327.

3 Tremblay LN, Slutsky AS. Ventilator-induced lung injury: from the bench to the bedside. Intensive Care Med 2006; 32: 24-33.

4 Diaz JV, Brower R, Calfee CS, et al. Therapeutic strategies for severe acute lung injury. Crit Care Med 2010; 38: 1644-1650.

5 Matthay M, Thompson BT, Read EJ, et al. Therapeutic potential of mesenchymal stem cells for severe acute lung injury. Chest 2010; 138: 965-972.

6 Abreu SC, Antunes MA, Pelosi P, et al. Mechanisms of cellular therapy in respiratory diseases. Intensive Care Med 2011; 37: 14211431.

7 Moodley Y, Manuelpillai U, Weiss DJ. Cellular therapies for lung disease: a distant horizon. Respirology 2011; 16: 223-237.

8 Koc ON, Day J, Nieder M, et al. Allogeneic mesenchymal stem cell infusion for treatment of metachromatic leukodystrophy (MLD) and Hurler syndrome (MPS-IH). Bone Marrow Transpl 2002; 30: 215-222.

9 Frank $\mathrm{MH}$, Sayegh $\mathrm{MH}$. Immunomodulatory functions of mesenchymal stem cells. Lancet 2004; 363: 1411-1412.

10 Rojas M, Xu J, Woods CR, et al. Bone marrow-derived mesenchymal stem cells in repair of the injured lung. Am J Respir Cell Mol Biol 2005; 33: 145-152.

11 Gupta N, Su X, Popov B, et al. Intrapulmonary delivery of bone marrow-derived mesenchymal stem cells improves survival and attenuates endotoxin-induced acute lung injury in mice. J Immunol 2007; 179: 1855-1863.

12 Prota LF, Lassance RM, Maron-Gutierrez T, et al. Bone marrow mononuclear cell therapy led to alveolar capillary membrane repair improving lung mechanics in endotoxin-induced acute lung injury. Cell Transplant 2010; 19: 965-971.

13 Krasnodembskaya A, Song Y, Fang X, et al. Antibacterial effect of human mesenchymal stem cells is mediated in part from secretion of the antimicrobial peptide LL-37. Stem Cells 2010; 28: 2229-2238. 
14 Lee JW, Fang X, Gupta N, et al. Allogeneic human mesenchymal stem cells for treatment of $E$. coli endotoxin-induced acute lung injury in the ex vivo perfused human lung. Proc Natl Acad Sci USA 2009; 106: 16357-16362.

15 Javazon EH, Colter DC, Schwarz EJ, et al. Rat marrow stromal cells are more sensitive to plating density and expand more rapidly from single-cell-derived colonies than human marrow stromal cells. Stem Cells 2001; 19: 219-225.

16 Danchuk S, Ylostalo JH, Hossain F, et al. Human multipotent stromal cells attenuate lipopolysaccharide-induced acute lung injury in mice via secretion of tumor necrosis factor-alpha-induced protein 6. Stem Cell Res Ther 2011; 2: 27.

17 Matute-Bello G, Downey G, Moore BB, et al. An official American Thoracic Society workshop report: features and measurements of experimental acute lung injury in animals. Am J Respir Cell Mol Biol 2011; 44: 725-738.

18 Vlahakis NE, Hubmayr RD. Cellular stress failure in ventilatorinjured lungs. Am J Respir Crit Care Med 2005; 171: 1328-1342.

19 Eyal FG, Hamm CR, Parker JC. Reduction in alveolar macrophages attenuates acute ventilator induced lung injury in rats. Intensive Care Med 2007; 33: 1212-1218.

20 Frank JA, Wray CM, McAuley DF, et al. Alveolar macrophages contribute to alveolar barrier dysfunction in ventilator-induced lung injury. Am J Physiol Lung Cell Mol Physiol 2006; 29: L1191-L1198.

21 Pugin J, Ricou B, Steinberg KP, et al. Proinflammatory activity in bronchoalveolar lavage fluids from patients with ARDS, a prominent role for interleukin-1. Am J Respir Crit Care Med 1996; 153: 1850-1856.

22 Gonzalez-Lopez A, Astudillo A, Garcia-Prieto E, et al. Inflammation and matrix remodeling during repair of ventilator-induced lung injury. Am J Physiol Lung Cell Mol Physiol 2011; 301: L500-L509.
23 Miyao N, Suzuki Y, Takeshita K, et al. Various adhesion molecules impair microvascular leukocyte kinetics in ventilator-induced lung injury. Am J Physiol Lung Cell Mol Physiol 2006; 290: L1059-L1068.

24 Carreras A, Almendros I, Acerbi I, et al. Obstructive apneas induce early release of mesenchymal stem cells into circulating blood. Sleep 2009; 32: 117-119.

25 Fischer UM, Harting MT, Jimenez F, et al. Pulmonary passage is a major obstacle for intravenous stem cell delivery: the pulmonary first-pass effect. Stem Cells Dev 2009; 18: 683-692.

26 Araujo IM, Abreu SC, Maron-Gutierrez T, et al. Bone marrowderived mononuclear cell therapy in experimental pulmonary and extrapulmonary acute lung injury. Crit Care Med 2010; 38: 1733-1741.

27 Abreu SC, Antunes MA, Maron-Gutierrez T, et al. Effects of bone marrow-derived mononuclear cells on airway and lung parenchyma remodeling in a murine model of chronic allergic inflammation. Respir Physiol Neurobiol 2010; 175: 153-163.

28 Aslam M, Baveja R, Liang OD, et al. Bone marrow stromal cells attenuate lung injury in a murine model of neonatal chronic lung disease. Am J Respir Crit Care Med 2009; 180: 1122-1130.

29 Wolthuis EK, Vlaar APJ, Choi G, et al. Mechanical ventilation using non-injurious ventilation settings causes lung injury in the absence of pre-existing lung injury in healthy mice. Critical Care 2009; 13: R1.

30 Ikegame Y, Yamashita K, Hayashi S, et al. Comparison of mesenchymal stem cells from adipose tissue and bone marrow for ischemic stroke therapy. Cytotherapy 2011; 13: 675-685.

31 Casteilla L, Planat-Benard V, Laharrague P, et al. Adipose-derived stromal cells: their identity and uses in clinical trials, an update. World J Stem Cells 2011; 3: 25-33. 